Vietnam Journal of Mechanics, VAST, Vol. 29, No. 3 (2007), pp. 285- 291

Special Issue Dedicated to the Memory of Prof. Nguyen Van Dao

\title{
A NOTE ON THE MELNIKOV FUNCTION
}

\author{
NGUYEN VAN DinH \\ Institute of Mechanics, VAST
}

\begin{abstract}
With one (Poincaré section) parameter and a particular motiom law (that associated to certain determined point of homo-heteroclinic orbits), the usual form of the Melnikov function seems to be not convenient for certain problems. Another favourable form can be obtained by using a supplementary parameter - the arbitrary time constant in the general mentioned motion law.
\end{abstract}

\section{INTRODUCTION}

As known, for Hamiltonian systems, homo-heteroclinic orbits (when they exist) can be destroyed by weak perturbations to generate - on Poincaré section - stable and unstable invariant manifolds and the distance between these manifolds can be estimated by the so-called Melnikov function ([1], pp. 356-389; [2], pp. 172-181). This distance is defined for manifolds on certain chosen Poincaré section and at certain determined point of the homo-heteroclinic orbits. Therefore, in the usual form of the Melnikov function, besides the parameter specifying the chosen Poincaré section, a particular motion law is used, that associated to the point of interest on the homo-heteroclinic orbits Varying the Poincaré section parameter, we can only study the variation of the mentioned distance on different Poincaré section at the same point of the homo-heteroclinic orbits. To study the variation of the distance between manifolds on a fixed Poincaré section and along the homo-heteroclinic orbits, we have to "modulate" the aforesaid motion law. This modulation can be realized by using a supplementary parameter as presented below.

\section{THE USUAL FORM OF THE MELNIKOV FUNCTION}

Let us consider weakly perturbed Hamiltonian systems governed by differential equations of the form:

$$
\ddot{x}=f(x)+\varepsilon g(x, t)
$$

or

$$
\begin{array}{ll}
\dot{x}_{1}=f_{1}\left(x_{1}, x_{2}\right)+\varepsilon g_{1}\left(x_{1}, x_{2}, t\right) ; & f_{1}\left(x_{1}, x_{2}\right)=\partial H\left(x_{1}, x_{2}\right) / \partial x_{2} \\
\dot{x}_{2}=f_{2}\left(x_{1}, x_{2}\right)+\varepsilon g_{2}\left(x_{1}, x_{2}, t\right) ; & f_{2}\left(x_{1}, x_{2}\right)=-\partial H\left(x_{1}, x_{2}\right) / \partial x_{1}
\end{array}
$$

where $x$ is a column vector variable with two components $\left(x_{1}, x_{2}\right) ; f$ and $g$ are column vector functions with two components $\left(f_{1}, f_{2}\right)$ and $\left(g_{1}, g_{2}\right)$, respectively; overdots denote differentiation with respect to time $t . g(x, t)$ represents a small perturbation $T$-periodic in $t ; H\left(x_{1}, x_{2}\right)$ is the Hamiltonian (a scalar-valued function) of the unperturbed system $(\varepsilon=0) ; \varepsilon$ is a small parameter. 
Assume that the unperturbed Hamiltonian system possesses an orbit $C$, homoclinic to a saddle 0 . This homoclinic orbit is destroyed by the small perturbation $\varepsilon g$ and on each Poincaré section $S_{\theta}$ specified by the parameter $\theta(0 \leqslant \theta<T)$, two invariant manifolds are generated: a stable manifold $C_{s}$ and an unstable one $C_{n}$; both these manifolds homoclinic to a new saddle $O^{\prime}$, close to $O$.

The distance between $C_{s}$ and $C_{n}$ is defined on certain chosen Poincaré section $S_{\theta}$ and at certain determined point $A$ of $C$. As known can be estimated by the so-called Melnikov function

$$
M(\theta)=\int_{-\infty}^{+\infty} f\left[x_{A}(t-\theta)\right] \wedge g\left[x_{A}(t-\theta), t\right] d t=\int_{-\infty}^{+\infty} f\left[x_{A}(\tau)\right] \wedge g\left[x_{A}(\tau), \tau+\theta\right] d \tau
$$

where $M(\theta)$ denotes the Melnikov estimation of the distance (along the normal of $C$ ) at $A$ between $C_{s}$ and $C_{n}$ on the Poincaré section $S_{\theta} ; \theta$ is the value of the parameter which specifies the chosen Poincaré section; $x_{A}(t)$ is the motion law (an unperturbed solution) associated to $A$ i.e. that satisfying the condition $x_{A}(0) \equiv A$ (thus, the representative point $x_{A}(t-\theta)$ moves along $C$ and passes through $A$ just at the moment $t=\theta$ ); the wedge symbol indicates the magnitude of the cross $(X)$ product (i.e. $f \wedge g=f_{1} g_{2}-f_{2} g_{1}$ ).

Fixing $A$ and varying $\theta,(2.3)$ gives - at the same (fixed) point $A$ of $C$ - the variation of the distance between $C_{s}$ and $C_{n}$ on different Poincaré sections. To study - on certain chosen (fixed) Poincaré section - the variation of the distance between $C_{s}, C_{n}$ along $C$, we have to fixe $\theta$ and "modulate" $x_{A}(t)$ i.e. each point $A$ needs its appropriate motion law.

In particular, to stydy on $S_{\theta}$ ( $\theta$-fixed, given) the distribution of intersection points of $C_{s}$ and $C_{n}$ along $C$, we have to solve the equation

$$
\int_{-\infty}^{+\infty} f\left[x_{A}(t-\theta)\right] \wedge g\left[x_{A}(t-\theta), t\right] d t=0
$$

where $x_{A}(t-\theta)$ is unknown motion law.

This problem becomes simple - as presented below - by introducing a new parameter $\alpha$ in $x_{A}(t)$.

\section{A MORE DETAILED FORM OF THE MELNIKOV FUNCTION}

We remark that the unperturbed Hamiltonian system $(\varepsilon=0)$ is autonomous. So, each of its solutions contains an arbitrary time constant i.e. if $x(t)$ is certain solution, $x(t+h)$ with arbitrary constant $h$ is also another solution of same orbit. In other words, corresponding to an unperturbed orbit, we have to deal with-not one-but a family of unperturbed solutions expressed by $x(t+h)$. We can consider $x(t)$ as a particular solution and $x(t+h)$ as the general solution along the aforesaid orbit (not the general solution of the system). Evidently, this remark can be applied to the special orbit - the homoclinic orbit $C$. Hence, when we find a particular motion law $x(t)$ along $C$, the general solution along $C$ is $x(t+h)$.

For the Melnikov function, it is convenient to write this general solution as

$$
x(t-\theta+\alpha)
$$


where $\theta$ is the Poincaré section parameter; $\alpha$ is the parameter attributed to the point $x(\alpha)=A_{\alpha} ; x(t)$ is the motion law associated to the point $A_{0}=x(0)$.

Using (3.1), the Melnikov function can be written as:

$$
\begin{aligned}
M(\theta, \alpha) & =\int_{-\infty}^{+\infty} f[x(t-\theta+\alpha)] \wedge g[x(t-\theta+\alpha), t] d t \\
& =\int_{-\infty}^{+\infty} f[x(\tau)] \wedge g[x(\tau), \tau+\theta-\alpha] d \tau
\end{aligned}
$$

$M(\theta, \alpha)$ is thus the distance at $A_{\alpha}=x(\alpha)$ between $C_{s}$ and $C_{n}$ on $S_{\theta}$. Fixing $\alpha$ and varying $\theta,(3.2)$ gives - at the same point $A_{\alpha}$ of $C$ - the distance between $C_{s}$ and $C_{n}$ on different Poincaré sections $S_{\theta}$. If, with $\theta$ fixed, we vary $\alpha,(3.2)$ gives the variation of the distance between $C_{s}$ and $C_{n}$ along $C$, on the already chosen (fixed) Poincaré section $S_{\theta}$. In particular, along $C$, the distribution of intersection points of $C_{s}$ and $C_{n}$ along $C$ on $S_{\theta}$ is determined by the equation:

$$
M(\theta, \alpha)=0
$$

where $\alpha$ is unknown and $\theta$ is constant.

Since $g(x, t)$ is $T$-periodic in $t, M(\theta, \alpha)$ is $T$-periodic in $\alpha$. Hence, when (3.3) admits a real root $\alpha$, it also admits an infinite number of roots $\alpha+2 n T$ ( $n$-integer). Correspondingly, we obtain along $C$, an infinite number of intersection points of $C_{s}$ and $C_{n}$; theses points are located near the points $A_{\alpha+2 n \pi}=x(\alpha+2 n \pi)$.

\section{AN EXAMPLE}

For illustration, we use the example 5.16 presented in ([1], pp. 374-377) i.e. we consider the externally forced Duffing oscillator governed by the differential equation

$$
\ddot{x}-x+x^{3}=\varepsilon\{p \cos (\Omega t)-k \dot{x}\} \quad \text { with } \quad \Omega=1
$$

where $x$ is a scalar variable, $p>0$ is intensity of the external forcing excitation $p \cos t$ of period $2 \pi ; k>0$ is the linear damping coefficient.

Putting $x=x_{1}, \dot{x}=x_{2}$, the equation (4.1) can be written as

$$
\begin{aligned}
& \dot{x}_{1}=x_{2}, \\
& \dot{x}_{2}=x_{1}-x_{1}^{3}+\varepsilon\left(p \cos t-k x_{2}\right) .
\end{aligned}
$$

Hence:

$$
\begin{aligned}
& f_{1}=x_{2}, f_{2}=x_{1}-x_{1}^{3}, g_{1}=0, g_{2}=p \cos t-k x_{2}, \\
& H(x, \dot{x})=H\left(x_{1}, x_{2}\right)=\frac{x_{1}^{2}}{2}+\frac{x_{1}^{4}}{4}-\frac{x_{1}^{2}}{2} .
\end{aligned}
$$

The unperturbed Hamiltonian system $(\varepsilon=0)$ possesses two homoclinic orbits - the right $C$ and the left $C^{\prime}$ - homoclinic to the saddle (origin) $O$, respectively encircling two centers $I(1,0)$ and $I^{\prime}(-1,0)$, intersecting the abscissa axis $O x \equiv O x_{1}$ at $A(\sqrt{2}, 0)$ and $A^{\prime}(-\sqrt{2}, 0)$. 
The equation of these orbits is

$$
H(x . \dot{x})=H\left(x_{1}, x_{2}\right)=0 \quad \text { or } \quad \dot{x}=x_{2}= \pm \sqrt{x_{1}^{2}-\frac{x_{1}^{4}}{2}}
$$

where $+(-)$ corresponds to upper (lower)-half homoclinic orbits, respectively.

The perturbation $\varepsilon\left(p \cos t-k x_{2}\right)$ destroys $C$ and $C^{\prime}$ to generate four invariant manifolds: the right and left unstable manifolds $C_{n}$ and $C_{n}^{\prime}$ and the right and left stable one $C_{s}$ and $C_{s}^{\prime}$.

Below, our study is devoted to the right homoclinic orbit $C$ and its corresponding stable and unstable invariant manifolds $C_{s}$ and $C_{n}$. A particular (unpertubed) motion law along $C$ is

$$
x(t)=x_{1}(t)=\sqrt{2} \operatorname{sech}(t), \quad \dot{x}(t)=x_{2}(t)=-\sqrt{2} \operatorname{sech}(t) \tanh (t) .
$$

For $t=0$, we have

$$
x_{1}(0)=\sqrt{2} \operatorname{sech}(0)=\sqrt{2}, \quad x_{2}(0)=-\sqrt{2} \operatorname{sech}(0) \tanh (0)=0 .
$$

Thus, the motion (4.6) is that associated to the point $A(\sqrt{2}, 0)$. The usual form of the Melnikov function is:

$$
M(\theta)=-\int_{-\infty}^{+\infty} \sqrt{2} \operatorname{sech}(t-\theta) \tanh (t-\theta)\{p \cos t+k \sqrt{2} \operatorname{sech}(t-\theta) \tanh (t-\theta)\} d t .
$$

It gives the estimation of the distance between $C_{s}$ and $C_{n}$ on the Poincaré section $S_{\theta}$ at the point $A(\sqrt{2}, 0)$. As in [1], if we vary $\theta$, we can study only the variation of the distance between $C_{s}$ and $C_{n}$ on different Poincaré sections $S_{\theta}$ at the same point $A$. The "general" motion law along $C$ is:

$$
x(t+\alpha)=\sqrt{2} \operatorname{sech}(t+\alpha), \quad \dot{x}(t+\alpha)=-\sqrt{2} \operatorname{sech}(t+\alpha) \tanh (t+\alpha)
$$

and the Melnikov function with two parameters is

$$
\begin{aligned}
M(\alpha, \theta)= & -\int_{-\infty}^{+\infty} \sqrt{2} \operatorname{sech}(t+\alpha-\theta) \tanh (t+\alpha-\theta) \times \\
& \times\{p \cos t+k \sqrt{2} \operatorname{sech}(t+\alpha-\theta) \tanh (t+\alpha-\theta)\} d t
\end{aligned}
$$

or

$$
M(\alpha, \theta)=-\int_{-\infty}^{+\infty} \sqrt{2} \operatorname{sech}(\tau) \tanh (\tau)\{p \cos (\tau+\theta-\alpha)+k \sqrt{2} \operatorname{sech}(\tau) \tanh (\tau)\} d \tau
$$

With (4.11), it is easy to estimate the distance between $C_{s}$ and $C_{n}$ on any Poincaré section $S_{\theta}$ and at any point of the homoclinic orbit $C$. For instance, at $A(\sqrt{2}, 0)$ and on $S_{0}, S_{\pi / 2}$ 
we obtain respectively

$$
\begin{aligned}
& M(\alpha=0, \theta=0)=-\sqrt{2} \int_{-\infty}^{+\infty} \operatorname{sech}(\tau) \tanh (\tau)\{p \cos \tau+k \sqrt{2} \operatorname{sech}(\tau) \tanh (\tau)\} d \tau \\
& M\left(\alpha=0, \theta=\frac{\pi}{2}\right)=-\sqrt{2} \int_{-\infty}^{+\infty} \operatorname{sech}(\tau) \tanh (\tau)\{-p \sin \tau+k \sqrt{2} \operatorname{sech}(\tau) \tanh (\tau)\} d \tau
\end{aligned}
$$

Let $B(1, \sqrt{2} / 2)$ be the maximum point of $C$. The value $\alpha_{*}$ corresponding to $B$ satisfies the equations:

$$
\sqrt{2} \operatorname{sech}\left(\alpha_{*}\right)=1, \quad-\sqrt{2} \operatorname{sech}\left(\alpha_{*}\right) \tanh \left(\alpha_{*}\right)=\frac{\sqrt{2}}{2} .
$$

Solving (4.11), we find $\alpha_{*}=\ln (\sqrt{2}-1) \approx-0.88137$. Therefore the distances at $B$ between $C_{s}$ and $C_{n}$ on $S_{0}, S_{\pi / 2}$ are estimated respectively by the integrals:

$$
\begin{aligned}
& M\left(\alpha_{*}, 0\right)=-\sqrt{2} \int_{-\infty}^{+\infty} \operatorname{sech}(\tau) \tanh (\tau)\left\{p \cos \left(\tau-\alpha_{*}\right)+k \sqrt{2} \operatorname{sech}(\tau) \tanh (\tau)\right\} d \tau \\
& M\left(\alpha_{*}, \frac{\pi}{2}\right)=-\sqrt{2} \int_{-\infty}^{+\infty} \operatorname{sech}(\tau) \tanh (\tau)\left\{-p \sin \left(\tau-\alpha_{*}\right)+k \sqrt{2} \operatorname{sech}(\tau) \tanh (\tau)\right\} d \tau
\end{aligned}
$$

Following the procedure given in [1], we expand (4.11) as:

$$
M(\alpha, \theta)=-k I_{1}-p \sqrt{2} \cos (\theta-\alpha) I_{2}+p \sqrt{2} \sin (\theta-\alpha) I_{3}
$$

where

$$
\begin{aligned}
I_{1} & =\int_{-\infty}^{+\infty} \dot{x}^{2}(\tau) d \tau, \quad I_{2}=\int_{-\infty}^{+\infty} \operatorname{sech}(\tau) \tanh (\tau) \cos \tau d \tau \\
I_{3} & =\int_{-\infty}^{+\infty} \operatorname{sech}(\tau) \tanh (\tau) \sin \tau d \tau
\end{aligned}
$$

Using (4.5), it is easy to calculate the first integral

$$
I_{1}=\int_{0}^{\sqrt{2}} \sqrt{x^{2}-x^{4} / 2} d x-\int_{\sqrt{2}}^{0} \sqrt{x^{2}-x^{4} / 2} d x=2 \int_{0}^{\sqrt{2}} \sqrt{x^{2}-x^{4} / 2} d x=\frac{4}{3} .
$$

The second integral $I_{2}=0$ because its integral is odd. 
Using $I_{2}=0$ and the transformations $u=e^{\tau}, v=e^{-\tau}$, the third integral can be put in the forms:

$$
\begin{aligned}
& I_{3}=\frac{1}{i} \int_{-\infty}^{+\infty} \operatorname{sech}(\tau) \tanh (\tau) e^{i \tau}=\frac{2}{i} \int_{0}^{\infty} u_{i} \frac{u^{2}-1}{\left(u^{2}+1\right)^{2}} d u, \\
& I_{3}=\frac{2}{i} \int_{-\infty}^{+\infty}(-1)^{i} v^{i} \frac{v^{2}-1}{\left(v^{2}+1\right)^{2}} d v=\frac{2}{i} e^{-\pi} \int_{-\infty}^{+\infty} v_{i} \frac{v^{2}-1}{\left(v^{2}+1\right)^{2}} d v
\end{aligned}
$$

Hence, we can write:

$$
I_{3}=\frac{2}{i\left(1+e^{-\pi}\right)} \int_{-\infty}^{+\infty} u^{2} \frac{u^{2}-1}{\left(u^{2}+1\right)^{2}} d u
$$

The integral (4.22) has a two folds singularity at $u=i$. Following the method of residues, we obtain

$$
I_{3}=\frac{2}{i\left(1+r^{-\pi}\right)} \cdot 2 \pi i \cdot \lim _{u \rightarrow i} \frac{d}{d u}\left\{(u-i)^{2} \frac{u^{2}\left(u^{2}-1\right)}{\left(u^{2}+1\right)^{2}}\right\}=\pi \operatorname{sech} \frac{\pi}{2}
$$

Finally:

$$
M(\alpha, \theta)=-\frac{4}{3} k+p \pi \sqrt{2} \sin (\theta-\alpha) \operatorname{sech} \frac{\pi}{2}
$$

Thus:

$$
\begin{aligned}
M(0,0) & =-\frac{4}{3} k ; \quad M\left(0, \frac{\pi}{2}\right)=-\frac{4}{3} k+p \pi \sqrt{2} \operatorname{sech} \frac{\pi}{2} \\
M\left(\alpha_{*}, 0\right) & =-\frac{4}{3} k+p \pi \sqrt{2} \sin \alpha_{*} \operatorname{sech} \frac{\pi}{2} \\
M\left(\alpha_{*}, \frac{\pi}{2}\right) & =-\frac{4}{3} k+p \pi \sqrt{2} \cos \alpha_{*} \operatorname{sech} \frac{\pi}{2}
\end{aligned}
$$

Using (4.24), we can easily study the distribution of intersection points of $C_{s}$ and $C_{n}$ along $C$ and - for intance - on $S_{0}$. Indeed, by solving the equation

$$
M(\alpha, \theta=0)=-\frac{4}{3} k-p \pi \sqrt{2} \sin \alpha \operatorname{sech} \frac{\pi}{2}=0
$$

we obtain

$$
\sin \alpha=-\frac{4 k}{3 p \pi \sqrt{2} \operatorname{sech} \frac{\pi}{2}}
$$

For $k \approx 0.5990222, \sin \alpha \approx-\sqrt{2} / 2, \alpha_{1}=-\frac{\pi}{4}+2 k \pi, \alpha_{2}=-\frac{3 \pi}{4}+2 k \pi$ corresponding to $\alpha_{1}$, we obtain:

- for $k=0,-1,-2, \ldots \quad x_{0}=1.0676459, x_{-1}=0.002408, x_{-2}=0.000004$

- for $k=1,2,3, \ldots \quad x_{1}=0.0115845, x_{2}=0.00000216, x_{3}=0.0000004$.

These are respectively the abscissae of the points of the upper and lower half-homoclinic orbit $C$ close intersection points of $C_{s}$ and $C_{n}$.

Similarly, from $\alpha_{2}$, we can immagine another infinite number of intersection points of $C_{s}$ and $C_{n}$, distributed along and close to $C$ and accumulated in the neighbourhood of $O$. 


\section{CONCLUSION}

From the above presented note, we see that, by introducing the time constant in the motion law i.e. by using the general motion law along homo-heteroclinic orbits, the Melnikov function can be put in a form containing two parameters and shows to be more convenient.

\section{REFERENCES}

1. A. H. Nayfeh, B. Balachandran, Applied Nonlinear Dynamics, John Wiley \& Sons, New-York, 1995.

2. J. Guckenheimer, P. Holmes, Nonlinear oscillations, Dynamical Systems and Bifurcations of Vector Fields, Springer, New York, 1986.

Received June 15, 2007.

\section{MỘT GHI CHÚ VỀ HÀM MELNIKOV}

Dạng quen biết của hàm Melnikov trong lý thuyết chuyển động hỗn loạn chỉ chứa một tham số xác định thiết diện Poincaré được lựa chọn. Nếu đưa thêm một tham số gán cho các điểm trên đường đồng dị clinic, có thể viết hàm Melnikov chứa hai tham số. Dạng sau tỏ ra thuận tiện hơn khi nghiên cứu sự phân bố giao điểm các đa tạp ởn định và không ổn định. 